III Sección: Educación, producción y finanzas

\title{
Greenwashing. Una apuesta riesgosa para el posicionamiento de una marca verde
}

Ana Sofia González Arredondo Tecnológico de Monterrey, Campus Monterrey, México ana.sofia.glzarr@gmail.com https://orcid.org/0000-0002-8023-7459 José Carlos Vázquez Parra Tecnológico de Monterrey, Campus Guadalajara, México jcvazquezp@tec.mx https://orcid.org/0000-0001-9197-7826

Recibido: 18 de agosto de 2020

Aceptado: 11 de noviembre de 2020

Resumen: Uno de los objetivos primordiales de una campaña de mercadotecnia es el poder posicionar o reposicionar un bien o servicio en la mente de sus consumidores. Sin embargo, ¿qué sucede cuando la técnica utilizada resulta ser percibida como una acción engañosa? El greenwashing es una técnica que algunas empresas han utilizado en los últimos años para promoverse como marcas verdes, aunque ello traiga consigo proponer acciones que no resultan coherentes con sus políticas de producción. Así, este artículo se orienta a hacer una aproximación teórica a la mercadotecnia verde y al greenwashing, buscando reflexionar acerca de los consumidores conscientes y cómo este tipo de acciones pueden resultar contraproducentes para las nuevas generaciones de compradores ya que las marcas son señaladas como incongruentes, afectando su reputación e imagen.

Palabras clave: Marketing; Mercado; Medio Ambiente; Responsabilidad Social; Comportamiento Económico.

Greenwashing. A risky bet for the positioning of a green brand.

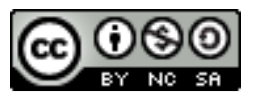

La Revista Estudios es editada por la Universidad de Costa Rica y se distribuye bajo una Licencia Creative Commons Atribución-NoComercial-CompartirIgual 3.0 Costa Rica. Para más información envíe un mensaje a 
Abstract: There is no doubt that one of the primary objectives of a marketing campaign is to position or reposition a good or service in the minds of its consumers. However, what happens when the technique used turns out to be perceived as a deceptive action? Greenwashing is a technique that some companies have used in recent years to consider themselves as green brands, even if this means proposing actions that are not consistent with their production policies. Thus, this article seeks to make a theoretical approach to green marketing and greenwashing, seeking to reflect on conscious consumers and how these types of actions can be counterproductive for the new generations of buyers when brands are pointed out as incongruent, affecting their reputation and image.

Keywords: Marketing; Market; Environment; Social Responsibility; Economic Behavior.

\section{Introducción}

Globalmente existen múltiples problemáticas ambientales (contaminación, deforestación, escasez de agua, extensión de biodiversidad, entre otros), y muchas de ellas, tienen relación con el consumo desmedido de las personas. Por ende, cada vez resulta mayor la tendencia, por parte de los consumidores, a desarrollar una consciencia colectiva, que tome en consideración los problemas, para poder participar a través de diferentes acciones en la búsqueda de soluciones que aporten a alcanzar una verdadera sostenibilidad. Nociones como la de consumo consciente, hace notorio el cada vez más marcado compromiso de las personas, quienes demandan de las empresas que consumen que se tomen acciones para poder mejorar su huella e impacto medioambiental. Así, las organizaciones contemporáneas, atendiendo a los reclamos de las nuevas generaciones, buscan apegarse a esta visión verde o eco-amigable, volviéndose una tendencia en los proyectos empresariales.

Sin embargo, es relevante que estas acciones no se enfoquen únicamente en promoverse como parte de un programa de captación de nuevos consumidores. Más que una ventaja competitiva, las acciones verdes deben plantearse de forma 
coherente en todos los procesos de producción y distribución de la empresa (ViteriLuque, Herrera-Lozano, Bazurto-Quiroz, 2017). Solo así la empresa podrá proyectar de manera efectiva que su comportamiento es real, mostrando un verdadero compromiso por generar un impacto positivo y una visión de responsabilidad social compartida.

Para alcanzar este objetivo de desarrollar proyectos verdes, pero a su vez, poder comunicarlos de forma efectiva, existe el llamado marketing verde. El marketing verde busca promover acciones medioambientales de producción y comercialización que llevan a cabo las organizaciones, desde un enfoque de generación de valor, mediante el cual, tanto organizaciones como consumidores, puedan reconocer su interés por este tipo de temas. En la actualidad, el marketing verde es una tendencia dentro de las campañas de comunicación empresarial, ya que se ha demostrado el gran impacto que puede llegar a tener como factor en la toma de decisiones de los consumidores.

Según Aguilar (2017), el marketing verde es una excelente oportunidad para las empresas, ya que significa diversos beneficios organizacionales, tanto por la promoción interna de interés hacia temas ambientales, como al constituirse como una estrategia de comunicación que permita posicionarse en nuevos mercados. En un estudio de Claudino y Talamini (2013) se calcula que cerca del $70 \%$ de los consumidores investigan sobre temas verdes y su relación con los productos que consumen, lo que demuestra un claro interés por ser parte de la solución y no del problema. Por otro lado, en un estudio de White, Hardisty y Habid (2019), se encontró que el $65 \%$ de los encuestados se sienten atraídos por productos que se producen de manera eco-amigable, aunque al momento de comprar, solo el $26 \%$ consiguen llevar las intenciones a una acción concreta.

En México la realidad no es diferente, ya que según Venegas (2019), 7 de cada 10 consumidores prefieren los productos verdes, y según una investigación de_Kantar

\section{(c) (i) (2)}

La Revista Estudios es editada por la Universidad de Costa Rica y se distribuye bajo una Licencia Creative Commons Atribución-NoComercial-CompartirIgual 3.0 Costa Rica. Para más información envíe un mensaje a 
ISSN 1659-3316

González Arredondo Ana Sofía

Vázquez Parra José Carlos

Worldpanel (2017), 1 de cada 4 están dispuestos a pagar más por aquellos productos que sean ecológicos o que de alguna manera apoyen el medio ambiente.

Por todo lo anterior, el presente artículo tiene el objetivo de hacer una aproximación a la mercadotecnia verde o greenmarketing como parte de los proyectos de comunicación empresarial. Se busca identificar, a partir de un análisis de casos, la necesidad de llevar a cabo acciones coherentes y un compromiso palpable, que eviten que las acciones se conviertan en una situación de greenwashing, que resulte ser contraproducente para la empresa y su marca. Para llegar a esto, primeramente, se hará una reflexión sobre el marketing verde y los llamados consumidores conscientes, para después reflexionar sobre algunos ejemplos concretos de acciones empresariales medioambientales que han sido calificadas como greenwashing y analizar cómo esta incongruencia entre políticas y compromiso ambiental tuvieron resultados contraproducentes que, en algunos casos, sigue afectando a dichas marcas (Marketing Directo, 2019).

\section{El marketing verde}

En términos generales, el marketing es un conjunto de actividades destinadas a satisfacer las necesidades y deseos de los mercados meta a cambio de una utilidad o beneficio para las empresas u organizaciones que la ponen en práctica; en ese sentido, el marketing es indispensable para lograr el éxito en los mercados actuales. El marketing es una disciplina que ha progresado en las últimas décadas, desarrollando nuevos enfoques que dan respuesta a las necesidades de los clientes actuales. Los consumidores cada día son más demandantes de información, tanto de los productos que consumen, como de las empresas que los generan. Así, se han generado nuevas tendencias de marketing, como pueden ser el marketing digital, el neuromarketing, el marketing personalizado y por supuesto, el marketing verde o ambiental.

\section{(C) $\odot \Theta \odot$}

La Revista Estudios es editada por la Universidad de Costa Rica y se distribuye bajo una Licencia Creative Commons Atribución-NoComercial-CompartirIgual 3.0 Costa Rica. Para más información envíe un mensaje a 
ISSN 1659-3316

González Arredondo Ana Sofía

Vázquez Parra José Carlos

Se entiende por marketing verde a aquellas acciones empresariales que buscan promover productos o servicios basándose en sus beneficios medioambientales. Este modelo también es conocido como comercialización verde, que a su vez propone cambios en la manera en que se comunican las empresas con sus consumidores. Para Novillo-Díaz, Pérez-Espinoza y Muñoz (2018), el marketing verde puede plantearse como una demanda por parte de los consumidores para que las empresas cuiden y preserven el medio ambiente a través de la producción y comercialización de sus productos y servicios, promoviendo que estos sean amigables con el medio ambiente. Según Peattie y Charter (2003), la modalidad de la mercadotecnia verde se puede clasificar en tres fases:

a. Marketing verde ecológico: La cual se desarrolló durante la década de los sesenta y setenta y se relacionaba con todas las actividades que buscaba resolver directamente problemas ambientales y cuyo fin era simplemente el de reconocer el rol que tenían las empresas, los productos y los consumidores ante estos problemas.

b. Marketing verde ambiental: Esta se dio en los años ochenta, y tenía el propósito de utilizar tecnología limpia en el diseño de productos innovadores y el mejoramiento del sistema de producción.

c. Marketing verde sostenible: Esta se dio a partir de los años 90, en la cual se buscaba atender la problemática de una forma más profunda, bajo una visión de sostenibilidad que no solo impactara en la producción, sino también en el consumo.

En la última etapa es donde se pone mayor atención a la relevancia que tiene el consumidor, como un eslabón clave en la promoción y sostenimiento de políticas verdes por parte de las empresas, dando cabida a nociones como el de consumidores conscientes o verdes. Para Sánchez-Castañeda (2014), un consumidor consciente es aquel que conoce y reconoce el impacto ambiental que

\section{(c) (†) (-)}

La Revista Estudios es editada por la Universidad de Costa Rica y se distribuye bajo una Licencia Creative Commons Atribución-NoComercial-CompartirIgual 3.0 Costa Rica. Para más información envíe un mensaje a 
tienen los productos y servicios que consume, considerando la forma en que sus hábitos pueden tener una relación positiva o negativa con la sostenibilidad ambiental. Según la organización civil Las Páginas Verdes (Connect Americas, 2018), el consumidor verde se define como una persona que manifiesta su preocupación por el medio ambiente, con base en su conducta de compra y buscando productos o servicios que sean percibidos de menor impacto sobre el medio ambiente.

Los perfiles del consumidor verde fueron identificados por Kantar Worldpanel (2018), en un estudio llamado GreenThermometer, el cual se basa en medir el grado de conciencia verde de los consumidores. En este estudio se identificaron 6 perfiles de consumidores

- El perfil " $360^{\circ}$ Green" es un consumidor que considera tres aspectos para su consumo: ambiental, social y económico. Este tipo de consumidor ve todo el panorama completo ya que las acciones verdes se vuelven hábitos y conductas en su estilo de vida, procura un impacto positivo ambiental y desperdiciar lo menos posible.

- El perfil "Green Society" es un consumidor que considera importante el aspecto social, sobre todo la solución de problemas sociales, así como la pobreza, hambre y educación. Este perfil con enfoque social se apoya en movimientos sociales y ONGs.

- El perfil "Green Shopper" es un consumidor que compra productos orgánicos y que, a su vez, buscan influenciar sobre las decisiones de los demás, enfocándose en un cambio positivo para el planeta.

- El perfil "Green Pocket" es un consumidor que busca beneficios económicos al momento de la toma de decisión de compra, cuidando el aspecto medio ambiental. Pueden tomar en cuenta servicios de agua y luz para la producción.

\section{(c) (i) (2)}

La Revista Estudios es editada por la Universidad de Costa Rica y se distribuye bajo una Licencia Creative Commons Atribución-NoComercial-CompartirIgual 3.0 Costa Rica. Para más información envíe un mensaje a revistaestudios.eeg@ucr.ac.cr. 
- El perfil "Dream Green" es un consumidor que sueña con el cambio positivo en el medio ambiente pero no precisamente toma acciones verdes. Están conscientes de que el cambio es necesario, pero se queda en una posición superficial.

- El perfil "Zero Green" es un consumidor que no tiene como prioridad el impacto positivo en el medio ambiente, y son ignorantes en cuanto al tema de la consciencia verde.

En cuanto a los factores que son relevantes para el consumidor verde, Monteiro, Giuliani, Cavazos-Arroyo y Kassouf-Pizzinatto (2015), presentan que los factores importantes son principalmente el respeto y el valor adicional que se tiene cuando un producto es ecológico. Estos factores influyen en el momento de elegir un bien o servicio por encima de otro con características semejantes.

Macías-Pérez (2020) explica la transición entre el consumidor verde y el consciente, a través del "efecto Greta", el cual habla de cómo la activista Greta Thunberg da a conocer que no es suficiente tomar acciones verdes, sino que también se debe desarrollar un panorama más completo sobre los problemas del medio ambiente. En este mismo estudio, Macías-Pérez (2020) señala que la noción de Emergencia Climática propuesta por Thunberg ha detonado una clara preocupación en los jóvenes sobre el rol y la responsabilidad que tienen sobre dicho problema. En el reporte de Marcas con Valores (2020) se encontró que "el 69\% de los ciudadanos con hijos menores de 20 años hace un consumo más consciente gracias a ellos” (p. 30), como lo señala Macías-Pérez (2020), son los jóvenes quienes mayor participación tienen en estos movimientos de concientización verde y son quienes mayores exigencias y demandas tienen hacia las empresas sobre estos temas.

Para los consumidores conscientes es importante toda la información de los productos que consumen al igual que las acciones por parte de la empresa. Es por esto que las empresas deben de aprovechar esta tendencia para tomar acciones,

\section{(c) (i) (2)}

La Revista Estudios es editada por la Universidad de Costa Rica y se distribuye bajo una Licencia Creative Commons Atribución-NoComercial-CompartirIgual 3.0 Costa Rica. Para más información envíe un mensaje a revistaestudios.eeg@ucr.ac.cr. 
pero sin dejar de ser congruente con el resto de sus políticas internas y externas, tanto de extracción, producción y distribución. De igual manera, en el reporte de Marcas con Valores (2020) se encontró que para la generación Z la autenticidad de una marca es un valor principal al momento de consumir un producto. Más allá de la publicidad, se busca que todas las acciones sean congruentes y, de no serlo, la imagen de la empresa puede ser fuertemente cuestionada.

Por todo lo anterior, muchas empresas han visto en el marketing verde una oportunidad para llamar la atención de nuevos mercados, enfocándose más en el mensaje que brindan, que en las acciones concretas que respaldan dichos proyectos de comunicación. Tras una careta de eco-amigables, plantean pocos o nulos cambios en sus productos, su cadena de suministros o sus líneas de producción, basándose más en la publicidad que en el compromiso. A lo anterior, se le conoce como greenwashing.

\section{El greenwashing}

Según García-Lombardía (2020), el concepto de "greenwashing" se define como una práctica que consiste en aprovechar el prestigio comercial de "lo verde" para obtener algún tipo de beneficio, sin considerar ese enfoque para el resto de sus acciones. Para Hallama, Montlló-Ribo, Rofas-Tudela y Ciutat-Vendrell (2011) el "greenwashing" se define como el uso por parte de una empresa u organización de una ampliación selectiva de la información medioambiental positiva, que produce una imagen distorsionada. Esta imagen se enfoca en demostrar la tendencia a favor de los aspectos y acciones "verdes", interpretados como positivos por los consumidores. Adicionalmente, para MacDonald (2019), "greenwashing" se define como una práctica poco ética de parte de las empresas y las marcas al crear una imagen de ser más ambientalmente responsable, de lo que son en realidad. En resumen, se puede concluir que un patrón común en estas definiciones es la falta

\section{(C) $(00$}

La Revista Estudios es editada por la Universidad de Costa Rica y se distribuye bajo una Licencia Creative Commons Atribución-NoComercial-CompartirIgual 3.0 Costa Rica. Para más información envíe un mensaje a 
de congruencia en cuanto a las acciones de una empresa y lo que comunica a sus consumidores.

La primera vez que se utilizó el concepto de greenwashing, conocido como lavado verde según Gutiérrez-Bonilla y Hernández-Lobato (2014), fue por el ecologista Jay Westervelt en un ensayo publicado en Nueva York en 1986 en relación con la industria hotelera. En el artículo destacó el término "lavado verde" debido a que la industria hotelera se publicitaba como socialmente responsable en el aspecto ambiental, al solicitar a sus huéspedes la reutilización de las toallas del hotel. Sin embargo, el objetivo de la campaña iba más hacia la ganancia publicitaria y económica, que al medio ambiente. Westervelt (Citado por Gutiérrez-Bonilla y Hernández-Lobato, 2014) recalca que, si la idea es causar un impacto positivo en el medio ambiente, se deben de considerar los aspectos que conllevan todas las acciones para que un hotel funcione, es decir, tomar en cuenta otros aspectos como la energía, el agua y emisiones y no simplemente el hecho de ahorrar un poco de agua en el lavado de las toallas de sus habitaciones.

Las empresas que utilizan el greenwashing son industrias que utilizan la tendencia del marketing verde para usarlo a su favor, como una ventaja competitiva e imagen de marca (Portugal-Mendiguri, 2019), cuando realmente no cumplen con los aspectos para ser considerado como eco-friendly. El greenwashing se puede presentar mediante empaques engañosos, etiquetas ecológicas falsas o frases mercadológicas como "producto 100\% natural", "producto orgánico" o "producto amigable con el medio ambiente".

\section{Casos de greenwashing}

A pesar de que el greenwashing ha sido una práctica que se ha llevado desde hace más de 30 años, hoy por hoy los consumidores se han vuelto más críticos y conscientes de su consumo, rechazando cualquier situación que pudiera ser engañosa o incongruente. Una empresa que esté publicitándose a través del 
ISSN 1659-3316

González Arredondo Ana Sofía

Vázquez Parra José Carlos

greenmarketing, que realmente acciona a través del greenwashing, es decir, que no tiene coherencia la publicidad y las acciones que se están tomando, puede caer en un escándalo, viendo afectada su imagen y sus relaciones públicas.

En muchos casos esto sucede con empresas que buscan ser consideradas socialmente responsables en temas medioambientales, y son descubiertas en una simulación de acciones verdes cuando la realidad es otra. En algunas ocasiones, a pesar de que la idea se propone con una intención positiva, puede haber complicaciones, ya que no se analiza la realidad de forma completa. Lo anterior puede afectar de manera negativa al medioambiente, ya sea en su producción, proceso o distribución. Es importante que las empresas busquen soluciones sustentables de acuerdo a sus políticas tanto internas como externas, viendo por todo el proceso y no únicamente por la pequeña parte que implica el consumo o el trato con los usuarios finales. Esta práctica de greenwashing se ve en todo tipo de empresas, organismos, marcas e incluso organizaciones gubernamentales.

\section{a. Volkswagen. Emisiones engañosas}

Uno de los ejemplos más sonados en los últimos años, debido a la magnitud del escándalo, es el de la empresa automotriz alemana Volkswagen. En el 2015, se descubrió que la empresa instaló un software que presentó información falsa en cuanto al número de emisiones contaminantes por parte de los vehículos de diésel creados por la empresa. Esta acción generó un costo negativo para la imagen de la empresa (Hotten, 2015). Además, por violar la Ley de Aire Limpio, se le asignó una multa que le costará a la compañía hasta 18,000 millones de dólares (Kleinman, 2016). Años después, este escándalo sigue afectando la imagen de esta industria automotriz. La reacción del público fue totalmente negativa debido a que se ignoraba desde cuando la empresa estaba tomando estas acciones (Plungis, 2015). En algunos artículos de blogs y redes sociales, los activistas refieren que este problema afectará varias décadas, otros mencionan los 11 millones de vehículos

\section{(c) (i) (2)}

La Revista Estudios es editada por la Universidad de Costa Rica y se distribuye bajo una Licencia Creative Commons Atribución-NoComercial-CompartirIgual 3.0 Costa Rica. Para más información envíe un mensaje a 
ISSN 1659-3316

González Arredondo Ana Sofía

Vázquez Parra José Carlos vendidos con estos reportes ambientales falsos (Partridge, 2015). En su momento el mercado cuestionó estas acciones, y declaró haberle perdido la confianza a esta marca automotriz.

\section{b. Nivea. Exfoliación plástica.}

Otro ejemplo de greenwashing fue el escándalo por parte de la empresa de productos de belleza y el cuidado personal, Nivea. Esta empresa, que propone ser $100 \%$ natural, ecológica y que cuida principalmente la sustentabilidad, lanzó una campaña enfocada en deshacerse de químicos tóxicos o parabenos en sus productos, como parte de la visión de la marca. Sin embargo, en el 2015, se descubrió a través de la organización medioambiental BUND que la empresa tenía una lista de productos (38, precisamente) con micro plásticos que afectaban al medio ambiente, primeramente, en sus exfoliantes (Aldred, 2016). La reacción del público fue totalmente negativa, la noticia provocó un escándalo debido a que se asumía que la empresa estaba enfocada 100\% tanto al cuidado facial como al cuidado del medio ambiente. Por si fuera poco, algunos dermatólogos afirmaron que el uso de estas micro partículas de plástico afectaban la piel facial y que, por ende, los productos de Nivea resultaban contraproducentes al objetivo que planteaban.

\section{c. Zara: Verde, pero socialmente irresponsable.}

En el mundo de la moda, un ejemplo de washing es el escándalo por parte de la industria de ropa "Fast Fashion", Zara. Aunque esta empresa española se hace llamar eco-friendly en cuanto al uso de energía y agua de sus tiendas, ha sido fuertemente cuestionada por los procesos laborales y de explotación al momento de la fabricación de sus prendas. En el documental "The True Cost", se critica a todo el Grupo Inditex, del cual forma parte Zara, por la violación de derechos laborales y explotación laboral a sus costureras, en sus diferentes fabricas alrededor del mundo (The Fashion Law, 2016). Actualmente, la industria de la moda rápida representa la venta de más de 80 billones de piezas de vestir al año, lo cual implica la necesidad

\section{(c) (i) (-)}

La Revista Estudios es editada por la Universidad de Costa Rica y se distribuye bajo una Licencia Creative Commons Atribución-NoComercial-CompartirIgual 3.0 Costa Rica. Para más información envíe un mensaje a 
de que los consumidores se mantengan muy activos en sus hábitos de compra. Lo anterior representa la adquisición de 4 veces más prendas que lo que se consumía hace 20 años (Frost, 2019). Empresas como Zara hacen lo laboralmente imposible para mantenerse al día en su oferta de prendas, aunque esto implique manejar un doble discurso acerca de su responsabilidad social.

\section{d. Starbucks: Bebidas plastificadas}

El café de Starbucks es preferido por muchos a lo largo del mundo entero, lo que le ha permitido tener más de 24,000 sucursales en 70 países. Aunque la marca de la sirena se preocupa por dar una imagen responsable a sus consumidores, esta visión se nubla cuando se reflexiona sobre los casi 250 billones de vasos de plástico de un solo uso que se utilizan cada año en sus bebidas (Lozanova, 2009). Por si esto fuera poco, su intento por reducir el consumo de popotes, los llevó a plantear una tapa que permite el consumo de sus productos directamente, sin embargo, al hacer un análisis de la misma, esta implica más plástico del que los popotes significaba. La principal defensa que ha usado la marca, es que usan solo plástico Bio-PBS, sin embargo, pocos son los países que cuentan con la tecnología para el reciclaje de este material, lo que lleva a que sus vasos terminen en la basura como cualquier otro desechable (Goddard, 2020).

\section{e. KFC: Contradicción en cada cubeta}

En la industria alimenticia, se puede considerar que una de las cadenas más representativas de la comida rápida es "Kentucky Fried Chicken”, también conocido como "KFC". Uno de los objetivos principales de la empresa es que tienen un compromiso con el cliente y con el medio ambiente lo cual resulta contradictorio. Desde 2010, las organizaciones como Dogwood Alliance, ForestEthics, Green America, Greenpeace US, the Gulf Restoration Network, Heartwood, NRDC, the Rainforest Action Network, y the Sierra Club (Butler, 2010), han denunciado los empaques de la marca como altamente contaminantes, sin tener ninguna respuesta 
o acción concreta por el gigante del pollo frito. Sin embargo, KFC plantea la posibilidad de hacer una pieza de pollo vegana, buscando ganar nuevos consumidores, aunque sus empaques sigan contaminando el mundo.

El tema del lavado verde, es un tema que se debe de considerar en la actualidad en todas las empresas. Es muy sencillo anunciar el hacer acciones verdes, pero debe de haber un compromiso para realmente llevarlas a cabo. Es importante recalcar que el uso de esta práctica puede causar efectos negativos en la imagen de la empresa, principalmente en cuanto a la desconfianza por parte de los consumidores conscientes, quienes han demostrado su capacidad de boicotear a aquellas marcas que engañen por medio de acciones contradictorias.

Según el informe de Marcas con Valor (2020), lo que se pone en juego con el greenwashing es la reputación de las empresas, lo cual llega a ser más dañino si las mismas se declaran inicialmente como socialmente responsables. Para la mercadotecnia, la imagen que da la empresa a sus clientes y la percepción que tienen sus consumidores de ella, resulta ser un aspecto clave al momento de la elección, compra y consumo de bienes y servicios. Basados en lo anterior, el ser amigablemente verde o socialmente responsable puede resultar ser determinante para las nuevas generaciones de consumidores.

\section{Conclusión}

Una vez analizado todo lo anterior, se puede señalar que la tendencia medioambiental se ha ido fortaleciendo conforme pasa el tiempo, sobre todo en las generaciones más jóvenes. La conciencia ecológica comienza a ser considerada en la toma de decisiones al momento de consumir cierto producto o servicio, lo que hace que las marcas deban poner mayor atención a la manera en que producen e impactan a su entorno. Es importante considerar que en la actualidad, el marketing verde es una tendencia dentro de las campañas de comunicación empresarial. Se ha demostrado el gran impacto que el marketing verde puede llegar a tener como

\section{(cc) (i) (9)}

La Revista Estudios es editada por la Universidad de Costa Rica y se distribuye bajo una Licencia Creative Commons Atribución-NoComercial-CompartirIgual 3.0 Costa Rica. Para más información envíe un mensaje a revistaestudios.eeg@ucr.ac.cr. 
factor en la toma de decisiones de los consumidores. A través de las acciones verdes se puede cambiar la perspectiva de una empresa, pero se debe de considerar con precaución las acciones que se llevarán a cabo para no caer en el lavado verde o greenwashing.

Las empresas requieren apegarse a la coherencia entre sus campañas de comunicación y sus acciones de producción, ya que, de no ser así, pueden ver afectada su reputación, resultando ser contraproducente su deseo de contar con una imagen verde. En el desarrollo de esta investigación se cumplió con el objetivo propuesto ya que se expusieron casos donde las empresas han tenido que actuar ante el greenwashing. Al igual, que la tendencia global de la concientización verde.

Es importante reconocer que los consumidores tienen el poder de presionar a las empresas a realizar acciones verdes o en su caso, tomar en cuenta las acciones que se estén realizando por medio de greenwashing. Los consumidores necesitan tomar esto en cuenta para poder actuar de manera contundente ante la problemática ambiental, lo cual empieza principalmente por los hábitos de consumo, las decisiones de compra y la adquisición de un producto/servicio. No es suficiente decir que se tiene un perfil como "Zero Green" o "Dream Green" donde la acción se queda únicamente como un deseo, pero realmente no se hace algo para cambiar las prácticas, o los aspectos que se consideran. El consumidor debe informarse acerca de las empresas de las cuales consume y hacer análisis de los productos que compra, manteniéndose al tanto, tomando acciones y buscando soluciones viables, si verdaderamente es consciente y comprometido.

Un área de oportunidad para nuevos estudios que deriven de este artículo es dar continuidad a los casos de greenwashing planteados, analizando el impacto económico que han tenido estos señalamientos de incongruencia, así como cuestionar el efecto en los hábitos de sus consumidores y la percepción de estos hacia la marca. A su vez, sería relevante considerar qué técnicas de comunicación 
ISSN 1659-3316

González Arredondo Ana Sofía

Vázquez Parra José Carlos

son necesarias para recuperarse de un escándalo mediático de greenwashing. Mientras las empresas sigan incurriendo en este tipo de prácticas, existe la necesidad de manejar estas crisis mediáticas y proponer estrategias para el reposicionamiento de una marca, una vez que ha fallado y engañado a sus consumidores.

Como se puede apreciar, tanto el greenwashing como la mercadotecnia verde son una realidad que los estrategas del marketing no pueden simplemente ignorar. Aunque resulta ser un tema complejo, puede ser determinante al momento de plantear una estrategia de comunicación, posicionamiento o reposicionamiento de una marca. Sin lugar a duda, la reputación de las empresas está en juego, y el greenwashing es una arriesgada apuesta.

\section{Referencias}

Aguilar, A. E. (2017). Marketing verde, una oportunidad para el cambio organizacional. Realidad y Reflexión, 16(44), p. 92-106. https://doi.org/10.5377/ryr.v44i0.3567

Aldred, J. (2016). Microplastics: Which beauty brands are safe to use? The Guardian. Recuperado de

https://www.theguardian.com/environment/2016/apr/19/microplastics-whichbeauty-brands-are-safe-to-use

Butler, R. (2010). Groups call on KFC to end greenwashing. Mongabay.com Recuperado de https://news.mongabay.com/2010/12/groups-call-on-kfc-to-endgreenwashing/

Claudino, E. S. y Talamini, E. (2013). Analise do Ciclo de Vida (ACV) aplicada a los agronegocio - Uma revisao de literatura. Revista Brasileira de Engenharia

\section{(C) $(\Theta \odot$}

La Revista Estudios es editada por la Universidad de Costa Rica y se distribuye bajo una Licencia Creative Commons Atribución-NoComercial-CompartirIgual 3.0 Costa Rica. Para más información envíe un mensaje a revistaestudios.eeg@ucr.ac.cr. 
Agricola e Ambiental, 17(1), p. 77-85. http://doi.org/10.1590/S141543662013000100011.

Connect Americas (2018) Organización con enfoque sustentable. Las páginas verdes. Connect Americas. Recuperado de https://connectamericas.com/es/company/las-paginas-verdes

Frost, R. (2019). Vogue features Zara's sustainability pledge, but is it greenwashing? Euro news. Recuperado de https://www.euronews.com/living/2019/07/17/vogue-feature-zara-ssustainability-pledge-but-is-it-greenwashing

García-Lombardía, P. (2020). 'Greenwashing' ser o no ser verde. Harvard Deusto Márketing y Ventas. Recuperado de https://www.harvarddeusto.com/greenwashing-ser-o-no-serverde\#: :text=M\%C3\%A1rketing\%20y\%20Ventas\%20(N\%C3\%BAm.\&text=El\% 20greenwashing\%20es\%20una\%20pr\%C3\%A1ctica,se\%20presume\%20es\%2 Overdaderamente\%20nimio.

Goddard, S. (2020). The Green Starbucks Cup: Green or Greenwashing? Green that life your guide to a sustainable lifestyle. Recuperado de https://greenthatlife.com/green-starbucks-cup-green-or-greenwashing/

Gutiérrez-Bonilla, R.E. y Hernández-Lobato, M. (2014). El desarrollo humano sostenible y los cambios que deberán realizar las empresas. Ciencia administrativa, (2). Recuperado de https://www.uv.mx/iiesca/files/2014/12/02CA201402.pdf

Hallama, M., Montlló-Ribo, M., Rofas-Tudela, S. y Ciutat-Vendrell, G. (2011). El fenómeno del greenwashing y su impacto sobre los consumidores propuesta

\section{(ब) $(\Theta \odot$}

La Revista Estudios es editada por la Universidad de Costa Rica y se distribuye bajo una Licencia Creative Commons Atribución-NoComercial-CompartirIgual 3.0 Costa Rica. Para más información envíe un mensaje a revistaestudios.eeg@ucr.ac.cr. 
ISSN 1659-3316

González Arredondo Ana Sofía Vázquez Parra José Carlos metodológica para su evaluación. Aposta. Revista de Ciencias Sociales, (50), p. 1-38.

Hotten, R. (2015). Volkswagen: The scandal explained. BBC News. Recuperado de https://www.bbc.com/news/business-34324772

Kantar Worldpanel (2017). 5 de junio: Día Mundial del Medio Ambiente. Kantar Worldpanel. Recuperado de https://www.kantarworldpanel.com/mx/Noticias-/5de-Junio-Dia-Mundial-del-Medio-Ambiente

Kantar Worldpanel (2018). Consumer Thermometer. Kantar Worldpanel. Recuperado de https://www.kantarworldpanel.com/mx/Noticias-/ConsumerThermometer-

Kleinman, D. (2016). Volkswagen podría enfrentar una multa de 18,000 mdd. Forbes México. Recuperado de https://www.forbes.com.mx/lo-que-una-multade-18000-mdd-a-vw-puede-dejarle-al-mundo/

Lozanova, S. (2009). Starbucks Coffee: Green or Greenwashed? GreenBiz. Recuperado de https://www.greenbiz.com/article/starbucks-coffee-green-orgreenwashed

MacDonald, S. (2019). Greenwashing de la imagen de las empresas cosméticas. Repositorio Comillas. Recuperado de https://repositorio.comillas.edu/xmlui/handle/11531/32674

Macías-Pérez, L. (2020). Efecto Greta: evolución de consumidor verde a consumidor consciente. Universidad Complutense de Madrid. Tesis de Grado en Administración y Dirección de Empresas. Recuperado de https://eprints.ucm.es/61485/

\section{(C) $\odot \odot \odot$}

La Revista Estudios es editada por la Universidad de Costa Rica y se distribuye bajo una Licencia Creative Commons Atribución-NoComercial-CompartirIgual 3.0 Costa Rica. Para más información envíe un mensaje a revistaestudios.eeg@ucr.ac.cr. 
Marcas con Valores (2020). III Estudio Marca con Valores. El poder del consumidor ciudadano. Ciudad de México: 21 gramos

Marketing Directo (2019). Greenwashing: No es verde todo lo que reluce. Marketing Directo.com. Recuperado de https://www.marketingdirecto.com/marketing-general/marketing/greenwashingno-es-verde-todo-lo-que-reluce

Monteiro, T. A., Giuliani, A. C., Cavazos-Arroyo, J., Kassouf-Pizzinatto, N. (2015). Mezcla del marketing verde: una perspectiva teórica. Cuadernos del CIMBAGE, (17), p. 103-116.

Novillo-Díaz, L. A., Pérez-Espinoza, M. J., \& Muñoz, J. C. (2018). Marketing verde, ¿tendencia o moda? Revista Universidad y Sociedad, 10(2), p. 100-105.

Partridge, K. (2015). VW Scandal Just the Tip of the Greenwashing Iceberg. Columbia Business School. Recuperado de https://www8.gsb.columbia.edu/articles/ideas-work/vw-scandal-just-tipgreenwashingiceberg\#: : :text=The\%20Volkswagen $\% 20$ scandal\%2C\%20deriving\%20from,the \%20reality\%20is\%20the\%20opposite.

Peattie, K., Charter, M. (2003). "Green Marketing” en: Baker Michael (ed.) The Marketing Book, p. 726-756. Oxford: Butterworth Heinemann

Plungis, J. (2015). Forty years of greenwashing: The well-travelled road taken by VW. Independent. Recuperado de https://www.independent.co.uk/news/business/analysis-andfeatures/volkswagen-emissions-scandal-forty-years-of-greenwashing-the-welltravelled-road-taken-by-vw-10516209.html

\section{(ब) $(\Theta \odot$}

La Revista Estudios es editada por la Universidad de Costa Rica y se distribuye bajo una Licencia Creative Commons Atribución-NoComercial-CompartirIgual 3.0 Costa Rica. Para más información envíe un mensaje a revistaestudios.eeg@ucr.ac.cr. 
ISSN 1659-3316

Diciembre 2020-Mayo 2021

González Arredondo Ana Sofía

Vázquez Parra José Carlos

Portugal-Mendiguri, M. D. (2019). Marketing verde y su relación con la imagen de marca. Grado académico de Bachiller en Ciencias Económico Empresariales. Recuperado de http://repositorio.ucsp.edu.pe/bitstream/UCSP/16031/1/PORTUGAL MENDIGU RI MEL MAR.pdf

Sánchez-Castañeda, J. (2014). Contextualización y enfoques en el estudio de comportamientos proambientales o ecológicos con miras a la perfilación del consumidor verde. Suma de Negocios, 5(10), p. 34-39. https://doi.org/10.1016/S2215-910X(14)70007-2

The Fashion Law (2016). Zara Boasts New Eco-Friendly Flagship, H\&M Focuses on Diversity. The Fashion Law. Recuperado de https://www.thefashionlaw.com/zara-open-new-eco-friendly-flagshipgreenwashing-much/

Venegas, E. (2019). 7 de cada 10 mexicanos opta por productos ecológicos. La Razón. Recuperado de https://www.razon.com.mx/negocios/7-de-cada-10mexicanos-opta-por-productos-ecologicos/

Viteri-Luque, F., Herrera-Lozano, L. A., y Bazurto-Quiroz, A. F. (2017). Las Tendencias del Marketing: Cuáles son y definiciones. RECIMUNDO: Revista Científica de la Investigación y el Conocimiento, 1(5), p. 974-988. https://doi.org/10.26820/recimundo/1.5.2017.974-988

White, K., Hardisty, D., \& Habib, R. (2019). The Elusive Green Consumer. Harvard Business Review, (7). Recuperado de https://hbr.org/2019/07/theelusive-green-consumer

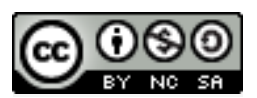

La Revista Estudios es editada por la Universidad de Costa Rica y se distribuye bajo una Licencia Creative Commons Atribución-NoComercial-CompartirIgual 3.0 Costa Rica. Para más información envíe un mensaje a revistaestudios.eeg@ucr.ac.cr. 